\title{
An Efficient Numerical Method for the Solution of the Schrödinger Equation
}

\author{
Licheng Zhang ${ }^{1}$ and Theodore E. Simos ${ }^{2,3}$ \\ ${ }^{1}$ School of Information Engineering, Chang'an University, Xi'an 710064, China \\ ${ }^{2}$ Department of Mathematics, College of Sciences, King Saud University, P.O. Box 2455, Riyadh 11451, Saudi Arabia \\ ${ }^{3}$ Laboratory of Computational Sciences, Department of Informatics and Telecommunications, Faculty of Economy, \\ Management and Informatics, University of Peloponnese, 22100 Tripolis, Greece
}

Correspondence should be addressed to Theodore E. Simos; tsimos.conf@gmail.com

Received 27 May 2016; Accepted 5 July 2016

Academic Editor: Maria L. Gandarias

Copyright (C) 2016 L. Zhang and T. E. Simos. This is an open access article distributed under the Creative Commons Attribution License, which permits unrestricted use, distribution, and reproduction in any medium, provided the original work is properly cited.

The development of a new five-stage symmetric two-step fourteenth-algebraic order method with vanished phase-lag and its first, second, and third derivatives is presented in this paper for the first time in the literature. More specifically we will study (1) the development of the new method, (2) the determination of the local truncation error (LTE) of the new method, (3) the local truncation error analysis which will be based on test equation which is the radial time independent Schrödinger equation, (4) the stability and the interval of periodicity analysis of the new developed method which will be based on a scalar test equation with frequency different than the frequency of the scalar test equation used for the phase-lag analysis, and (5) the efficiency of the new obtained method based on its application to the coupled Schrödinger equations.

\section{Introduction}

The approximate solution of the close-coupling differential equations of the Schrödinger type is studied in this paper. The above-mentioned problem has the following form:

$$
\left[\frac{d^{2}}{d x^{2}}+k_{i}^{2}-\frac{l_{i}\left(l_{i}+1\right)}{x^{2}}-V_{i i}\right] y_{i j}=\sum_{m=1}^{N} V_{i m} y_{m j}
$$

where $1 \leq i \leq N$ and $m \neq i$ and the boundary conditions are as follows:

$$
\begin{aligned}
& y_{i j}=0 \quad \text { at } x=0 \\
& y_{i j} \sim k_{i} x j_{l_{i}}\left(k_{i} x\right) \delta_{i j}+\left(\frac{k_{i}}{k_{j}}\right)^{1 / 2} K_{i j} k_{i} x n_{l i}\left(k_{i} x\right),
\end{aligned}
$$

where $j_{l}(x)$ and $n_{l}(x)$ are the spherical Bessel and Neumann functions. We will examine the case in which all channels are open (see [1]). [1])

Defining a matrix $K^{\prime}$ and diagonal matrices $M, N$ by (see

$$
\begin{aligned}
K_{i j}^{\prime} & =\left(\frac{k_{i}}{k_{j}}\right)^{1 / 2} K_{i j}, \\
M_{i j} & =k_{i} x j_{l_{i}}\left(k_{i} x\right) \delta_{i j}, \\
N_{i j} & =k_{i} x n_{l_{i}}\left(k_{i} x\right) \delta_{i j}
\end{aligned}
$$

we obtain a new form of the asymptotic condition (3):

$$
\mathbf{y} \sim \mathbf{M}+\mathbf{N K}^{\prime} .
$$

In several scientific areas (e.g., quantum chemistry, theoretical physics, material science, atomic physics, and molecular physics) there exists a real problem which is the rotational excitation of a diatomic molecule by neutral particle impact. The mathematical model of this problem can be expressed with close-coupling differential equations arising from the Schrödinger equation. Denoting, as in [1], 
the entrance channel by the quantum numbers $(j, l)$, the exit channels by $\left(j^{\prime}, l^{\prime}\right)$, and the total angular momentum by $J=$ $j+l=j^{\prime}+l^{\prime}$, we find that

$$
\begin{aligned}
& {\left[\frac{d^{2}}{d x^{2}}+k_{j^{\prime} j}^{2}-\frac{l^{\prime}\left(l^{\prime}+1\right)}{x^{2}}\right] y_{j^{\prime} l^{\prime}}^{J j l}(x)} \\
& \quad=\frac{2 \mu}{\hbar^{2}} \sum_{j^{\prime \prime}} \sum_{l^{\prime \prime}}\left\langle j^{\prime} l^{\prime} ; J|V| j^{\prime \prime} l^{\prime \prime} ; J\right\rangle y_{j^{\prime \prime} l^{\prime \prime}}^{J j l}(x),
\end{aligned}
$$

where

$$
k_{j^{\prime} j}=\frac{2 \mu}{\hbar^{2}}\left[E+\frac{\hbar^{2}}{2 I}\left\{j(j+1)-j^{\prime}\left(j^{\prime}+1\right)\right\}\right] .
$$

$E$ is the kinetic energy of the incident particle in the centerof-mass system, $I$ is the moment of inertia of the rotator, and $\mu$ is the reduced mass of the system.

The above-described problem will be solved numerically via finite difference method of the form of special multistep method. form

The multistep finite difference method has the general

$$
\sum_{i=-m}^{m} c_{i} y_{n+i}=h^{2} \sum_{i=-m}^{m} b_{i} f\left(x_{n+i}, y_{n+i}\right)
$$

where $\left\{x_{i}\right\}_{i=-m}^{m}$ are distinct points within the integration area and $h$ given by $h=\left|x_{i+1}-x_{i}\right|, i=1-m(1) m-1$ is the step size or step length of the integration.

Remark 1. A method (8) is called symmetric multistep method or symmetric $2 m$-step method if $c_{-i}=c_{i}$ and $b_{-i}=$ $b_{i}, i=0(1) m$.

If we apply the symmetric $2 m$-step method $(i=-m(1) m)$, to the scalar test equation

$$
y^{\prime \prime}=-\phi^{2} y
$$

we obtain the difference equation

$$
\begin{array}{r}
A_{m}(v) y_{n+m}+\cdots+A_{1}(v) y_{n+1}+A_{0}(v) y_{n} \\
+A_{1}(v) y_{n-1}+\cdots+A_{m}(v) y_{n-m}=0
\end{array}
$$

and the associated characteristic equation

$$
\begin{gathered}
A_{m}(v) \lambda^{m}+\cdots+A_{1}(v) \lambda+A_{0}(v)+A_{1}(v) \lambda^{-1}+\cdots \\
+A_{m}(v) \lambda^{-m}=0
\end{gathered}
$$

where $v=\phi h, h$ is the step length, and $A_{j}(v) j=0(1) k$ are polynomials of $v$.

We give some definitions.

Definition 2 (see [2]). For a symmetric $2 m$-step method with characteristic equation given by (11) one will say that it has an interval of periodicity $\left(0, v_{0}^{2}\right)$ if, for all $v \in\left(0, v_{0}^{2}\right)$, the roots $\lambda_{i}, \quad i=1(1) 2 m$, of (11) satisfy

$$
\begin{aligned}
\lambda_{1} & =e^{i \theta(v)}, \\
\lambda_{2} & =e^{-i \theta(v)}, \\
\left|\lambda_{i}\right| & \leq 1, \quad i=3(1) 2 m,
\end{aligned}
$$

where $\theta(v)$ is a real function of $v$.

Definition 3 (see [2]). One calls P-stable method a multistep method if its interval of periodicity is equal to $(0, \infty)$.

Definition 4. One calls singularly P-stable method a multistep method if its interval of periodicity is equal to $(0, \infty)-S$ (where $S$ is a set of distinct points).

Definition 5 (see $[3,4]$ ). For a symmetric $2 m$-step method with the characteristic equation given by (11), one defines as the phase-lag the leading term in the expansion of

$$
t=v-\theta(v) .
$$

Then, if the quantity $t=O\left(v^{q+1}\right)$ as $v \rightarrow \infty$, the order of the phase-lag is $q$.

Definition 6 (see [5]). One calls symmetric $2 m$-step method phase-fitted if its phase-lag is equal to zero.

Theorem 7 (see [3]). The symmetric 2 m-step method with characteristic equation given by (11) has phase-lag order $q$ and phase-lag constant c given by

$$
\begin{aligned}
& -c v^{q+2}+O\left(v^{q+4}\right) \\
& =\frac{2 A_{m}(v) \cos (m v)+\cdots+2 A_{j}(v) \cos (j v)+\cdots+A_{0}(v)}{2 m^{2} A_{m}(v)+\cdots+2 j^{2} A_{j}(v)+\cdots+2 A_{1}(v)} .
\end{aligned}
$$

\section{The New Five-Stage Fourteenth-Algebraic Order P-Stable Two-Step Method with Vanished Phase-Lag and Its First, Second, and Third Derivatives}

We consider the following family of five-stage symmetric two-step methods:

$$
\begin{aligned}
& \widehat{y}_{n}=y_{n}-a_{0} h^{2}\left(f_{n+1}-2 f_{n}+f_{n-1}\right), \\
& \check{y}_{n}=y_{n}-a_{1} h^{2}\left(f_{n+1}-2 \widehat{f}_{n}+f_{n-1}\right)-2 a_{2} h^{2} \widehat{f}_{n}, \\
& \widehat{y}_{n+1 / 2}=\frac{1}{2}\left(y_{n}+y_{n+1}\right)-h^{2}\left[a_{3} \check{f}_{n}+\left(\frac{1}{8}-a_{3}\right) f_{n+1}\right], \\
& \widehat{y}_{n-1 / 2}=\frac{1}{2}\left(y_{n}+y_{n-1}\right)-h^{2}\left[a_{3} \check{f}_{n}+\left(\frac{1}{8}-a_{3}\right) f_{n-1}\right], \\
& y_{n+1}+a_{4} y_{n}+y_{n-1} \\
& =h^{2}\left[b_{1}\left(f_{n+1}+f_{n-1}\right)+b_{0} f_{n}+b_{2}\left(\widehat{f}_{n+1 / 2}+\widehat{f}_{n-1 / 2}\right)\right],
\end{aligned}
$$


where $a_{0}=45469 / 862066800, a_{1}=-2793 / 26878564, a_{2}=$ $-86919 / 13439282, a_{3}=6719641 / 52720800, f_{n+i}=y^{\prime \prime}\left(x_{n+i}\right.$, $\left.y_{n+i}\right), i=-1(1 / 2) 1, \widehat{f}_{n}=y^{\prime \prime}\left(x_{n}, \widehat{y}_{n}\right), \check{f}_{n}=y^{\prime \prime}\left(x_{n}, \breve{y}_{n}\right)$, and $a_{4}, b_{j}, j=0(1) 2$, are free parameters.

Application of the above-mentioned method (15) to the scalar test equation (9) leads to the difference equation (10) and the characteristic equation (11) with $m=1$ and

$$
\begin{aligned}
A_{1}(v)= & 1+v^{2}\left(b_{1}+\frac{1}{2} b_{2}\right)-v^{4} b_{2}\left(a_{3}-\frac{1}{8}\right) \\
& +2 v^{6} b_{2} a_{1} a_{3}+4 v^{8} b_{2} a_{0} a_{3}\left(a_{2}-a_{1}\right), \\
A_{0}(v)= & a_{4}+v^{2}\left(b_{0}+b_{2}\right)+2 v^{4} b_{2} a_{3} \\
& +4 v^{6} b_{2} a_{3}\left(a_{2}-a_{1}\right) \\
& -8 v^{8} b_{2} a_{0} a_{3}\left(a_{2}-a_{1}\right) .
\end{aligned}
$$

If we request the new method (15) to have vanished phase-lag and its first, second, and third derivatives, then the following system of equations is obtained:

$$
\text { Phase-Lag }(\mathrm{PL})=\frac{T_{0}}{T_{\text {denom }}}=0 \text {, }
$$

First Derivative of the Phase-Lag $=\frac{T_{1}}{T_{\text {denom }}^{2}}=0$,

Second Derivative of the Phase-Lag $=\frac{T_{2}}{T_{\text {denom }}^{3}}=0$,

Third Derivative of the Phase-Lag $=\frac{T_{3}}{T_{\text {denom }}^{4}}=0$,

where $T_{j}, j=0(1) 3$, are given in Appendix A.

Now solving the system of (17) we determine the other coefficients of the new obtained three-stage two-step method:

$$
\begin{aligned}
& b_{0}=\frac{1}{136407} \frac{T_{4}}{T_{\text {denom } 1}}, \\
& b_{1}=\frac{1}{136407} \frac{T_{5}}{T_{\text {denom } 1}}, \\
& b_{2}=\frac{1}{45469} \frac{T_{6}}{T_{\text {denom } 1}}, \\
& a_{4}=\frac{T_{7}}{T_{\text {denom } 2}},
\end{aligned}
$$

where the formulae $T_{j}, j=4(1) 7, T_{\text {denom1 }}$, and $T_{\text {denom2 }}$ are given in Appendix $B$.

Additionally to the above formulae for the coefficients $a_{4}, b_{j}, j=0(1) 2$, we give also the following Taylor series expansions of these coefficients, for the case of heavy cancelations for some values of $|v|$ in the formulae given by (18):

$b_{0}$

$$
\begin{aligned}
= & \frac{73205}{63882}+\frac{268231049 v^{10}}{262060331977892448} \\
& -\frac{473946402833 v^{12}}{224533292438658249446400} \\
& -\frac{841146961608850447 v^{14}}{26876635104907392458734080000} \\
& -\frac{34711252155550520483 v^{16}}{23840650403457053406595478323200} \\
& -\frac{450175574700292070703419 v^{18}}{7016973932030008047188109768345600000} \\
& +\cdots,
\end{aligned}
$$

$b_{1}$

$$
\begin{aligned}
= & \frac{51911}{383292}+\frac{268231049 v^{10}}{1572361991867354688} \\
& -\frac{61738277963993 v^{12}}{1347199754631949496678400} \\
& -\frac{559868876269811659 v^{14}}{161259810629444354752404480000} \\
& -\frac{602760578937670659833 v^{16}}{3576097560518558010989321748480000} \\
& -\frac{526438577422138492034251 v^{18}}{84203687184360096566257317220147200000} \\
& +\cdots,
\end{aligned}
$$

$b_{2}$

$$
\begin{aligned}
= & -\frac{19970}{95823}-\frac{268231049 v^{10}}{393090497966838672} \\
& +\frac{15790029293123 v^{12}}{336799938657987374169600} \\
& +\frac{181541151070183 v^{14}}{40314952657361088688101120} \\
& +\frac{21664254981643052111 v^{16}}{89402439012963950274733043712000} \\
& +\frac{8364955394669318397937 v^{18}}{842036871843600965662573172201472000} \\
& +\cdots,
\end{aligned}
$$$$
a_{4}
$$

$$
\begin{aligned}
= & -2+\frac{134317 v^{16}}{15752663892725760} \\
& +\frac{1170023347 v^{18}}{4217775757277322240000}+\cdots .
\end{aligned}
$$

The behavior of the coefficients is given in Figure 1. 

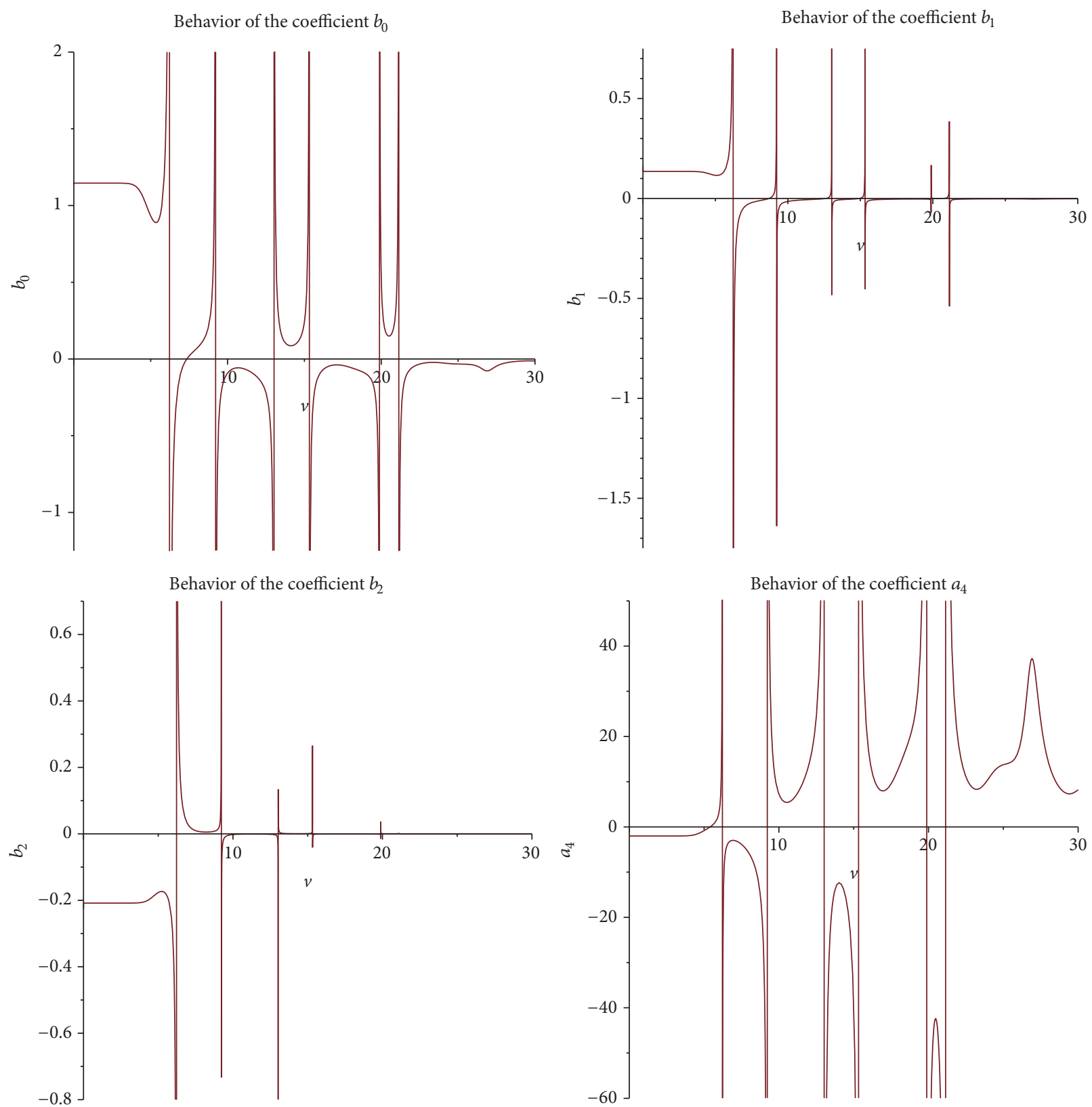

FIGURE 1: Behavior of the coefficients of the new obtained method given by (18) for several values of $v=\phi h$.

The local truncation error of the new developed five-stage two-step method (15) with the coefficients given by (18)-(19), which is indicated as NM2S5S3DV, is given by

$$
\begin{aligned}
& \mathrm{LTE}_{\mathrm{NM} 2 S 5 S 3 \mathrm{DV}}=\frac{134317}{551343236245401600} h^{16}\left(y_{n}^{(16)}\right. \\
& \left.\quad+56 \phi^{10} y_{n}^{(6)}+140 \phi^{12} y_{n}^{(4)}+120 \phi^{14} y_{n}^{(2)}+35 \phi^{16} y_{n}\right) \\
& \quad+O\left(h^{18}\right) .
\end{aligned}
$$

\section{Analysis of the Method}

3.1. Error Analysis. The test equation used for the local truncation error (LTE) analysis is given by

$$
y^{\prime \prime}(x)=\left(V(x)-V_{c}+G\right) y(x),
$$

where $V(x)$ is a potential function, $V_{c}$ is a constant value approximation of the potential for the specific $x$, and $G=$ $V_{c}-E$ and $E$ is the energy. This is the radial time independent Schrödinger equation.

We will study the following methods.

3.1.1. Classical Method (i.e., Method (15) with Constant Coefficients)

$$
\mathrm{LTE}_{\mathrm{CL}}=\frac{134317}{551343236245401600} h^{16} y_{n}^{(16)}+O\left(h^{18}\right) .
$$


3.1.2. The Five-Stage Two-Step Method with Vanished

Phase-Lag and Its First, Second, and

Third Derivatives Developed in Section 3

$$
\begin{aligned}
& \mathrm{LTE}_{\mathrm{NM} 255 \mathrm{~S} 3 \mathrm{DV}}=\frac{134317}{551343236245401600} h^{16}\left(y_{n}^{(16)}\right. \\
& \left.\quad+56 \phi^{10} y_{n}^{(6)}+140 \phi^{12} y_{n}^{(4)}+120 \phi^{14} y_{n}^{(2)}+35 \phi^{16} y_{n}\right) \\
& \quad+O\left(h^{18}\right) .
\end{aligned}
$$

If we substitute the higher order derivatives requested in the LTE formulae, which are obtained using the test problem (21), into the LTE expressions, we produce new formulae of LTE which have the general form

$$
\mathrm{LTE}=h^{p} \sum_{i=0}^{m} a_{i} G^{i},
$$

where $a_{i}$ are constant numbers (classical methods) or formulae of $\phi$ (fitted methods) and $p$ is the algebraic order of the specific method.

Two cases of the parameter $G$ are studied:

(i) The Energy and the Potential Are Closed to Each Other. Consequently, $G=V_{c}-E \approx 0 \Rightarrow G^{i}=0, i=1,2, \ldots$.

Therefore, the local truncation error for the classical method (constant coefficients) and the local truncation error for the five-stage two-step method with eliminated phase-lag and its first, second, and third derivatives developed in Section 3 are the same since the formulae of the LTE are free from $G$ (i.e., LTE = $h^{p} a_{0}$ in (24)) and the free from $G$ terms (i.e., the terms of the formulae which do not have the quantity $G$ ) in the local truncation errors in this case are the same and are given in Appendix C. From the above it is easy to see that, for these values of $G$, the methods are of comparable accuracy.

(ii) The Energy and the Potential Have Big Difference. Consequently, $G \gg 0$ or $G \ll 0$ and $|G|$ is a large number. For this case the most accurate method is the method with the minimum power of $G$ in the formulae of LTE (i.e., the method with the highest accuracy is the method with minimum $i$ in (24)).

We give now the asymptotic expansions of the local truncation errors.

\subsubsection{Classical Method}

$$
\begin{aligned}
\operatorname{LTE}_{\mathrm{CL}}= & \frac{134317}{551343236245401600} h^{16}\left(y(x) G^{8}+\cdots\right) \\
& +O\left(h^{18}\right) .
\end{aligned}
$$

3.1.4. The Four-Stage Two-Step P-Stable Method with Vanished Phase-Lag and Its First, Second, and Third Derivatives Developed in Section 3

$$
\begin{aligned}
& \mathrm{LTE}_{\mathrm{NM} 2553 \mathrm{DV}}=\frac{134317}{9845414932953600} \\
& \cdot h^{16}\left[\left(20 g(x) y(x) \frac{d^{2}}{d x^{2}} g(x)\right.\right. \\
& +15\left(\frac{d}{d x} g(x)\right)^{2} y(x) \\
& +10\left(\frac{d^{3}}{d x^{3}} g(x)\right) \frac{d}{d x} y(x) \\
& \left.\left.+31\left(\frac{d^{4}}{d x^{4}} g(x)\right) y(x)\right) G^{5}+\cdots\right]+O\left(h^{18}\right) .
\end{aligned}
$$

The above leads us to the following theorem.

Theorem 8. (i) Classical method (i.e., method (15) with constant coefficients): for this method the error increases as the eighth power of $G$.

(ii) Fourteenth-algebraic order five-stage two-step method with vanished phase-lag and its first, second, and third derivatives developed in Section 3: for this method the error increases as the fifth power of G.

Therefore, for large values of $|G|=\left|V_{c}-E\right|$, the new developed fourteenth-algebraic order five-stage two-step method with vanished phase-lag and its first, second, and third derivatives developed in Section 3 is the most accurate method for the numerical solution of the radial Schrödinger equation.

3.2. Stability and Interval of Periodicity Analysis. Let us define the scalar test equation for the stability and interval of periodicity analysis:

$$
y^{\prime \prime}=-\omega^{2} y
$$

Remark 9. Comparing the test equations (9) and (27), we have that the frequency $\phi$ is not equal to the frequency $\omega$; that is, $\omega \neq \phi$.

The difference equation which is produced after application of the new method (15) with the coefficients given by (18)-(19) to the scalar test equation (27) is given by

$$
A_{1}(s, v)\left(y_{n+1}+y_{n-1}\right)+A_{0}(s, v) y_{n}=0,
$$

where

$$
\begin{aligned}
A_{1}(s, v)=1 & +\frac{1}{8}\left(8 b_{1}+4 b_{2}\right) s^{2}+\frac{1}{8} b_{2}\left(-8 a_{3}+1\right) s^{4} \\
& +4 a_{3} b_{2}\left(v^{2} a_{0} a_{2}+\frac{1}{2} a_{1}\right) s^{6} \\
& -4 a_{0} a_{1} a_{3} b_{2} s^{8} \\
A_{0}(s, v)= & a_{4}+\left(b_{0}+b_{2}\right) s^{2}+4 a_{3} b_{2}\left(v^{2} a_{2}+\frac{1}{2}\right) s^{4}
\end{aligned}
$$




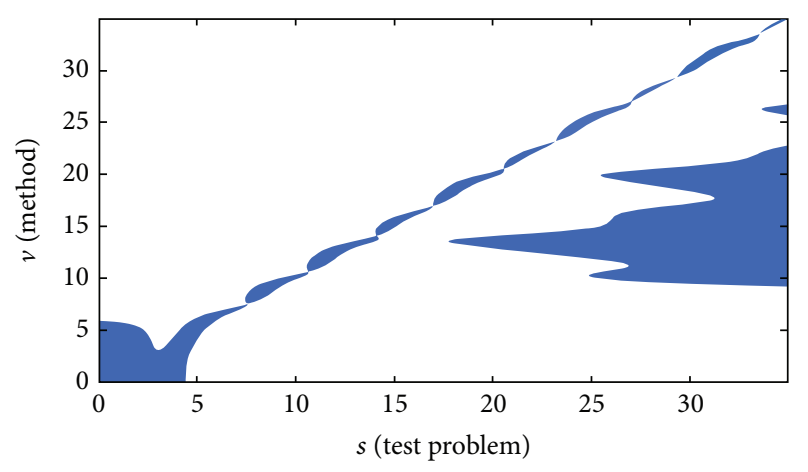

FIGURE 2: $s-v$ plane of the new obtained five-stage symmetric twostep fourteenth-algebraic order method with vanished phase-lag and its first, second, and third derivatives.

$$
\begin{aligned}
& -8 a_{3} b_{2}\left(v^{2} a_{0} a_{2}+\frac{1}{2} a_{1}\right) s^{6} \\
& +8 a_{0} a_{1} a_{3} b_{2} s^{8}
\end{aligned}
$$

$s=\omega h$, and $v=\phi h$.

Taking into account the coefficients $a_{i}, i=0,1$, and $b_{i}, i=0(1) 2$, and their substitution into formulae (29), the new stability polynomials are produced:

$$
\begin{aligned}
& A_{1}(s, v)=\frac{T_{8}}{T_{\text {denom } 3}}, \\
& A_{0}(s, v)=\frac{T_{9}}{T_{\text {denom } 3}},
\end{aligned}
$$

where the formulae $T_{k}, k=8,9$, and $T_{\text {denom3 }}$ are given in Appendix D.

$s-v$ plane of the new produced method is shown in Figure 2 .

Remark 10. Observing $s-v$ region we can define two areas:

(i) The shadowed area which defines the area where the method is stable.

(ii) The white area which defines the area where the method is unstable.

For problems of the form of the coupled equations arising from the Schrödinger equation and related problems (in quantum chemistry, material science, theoretical physics, atomic physics, astronomy, astrophysics, physical chemistry, and chemical physics), the area of the region which plays critical role in the stability of the numerical methods is the surroundings of the first diagonal of the $s-v$ plane, where $s=$ $v$. Studying this case, we found that the interval of periodicity is equal to $(0,24)$.

The above development leads to the following theorem.

Theorem 11. The five-stage symmetric two-step method developed in Section 3

(i) is of fourteenth algebraic order, (ii) has vanished the phase-lag and its first, second, and third derivatives,

(iii) has an interval of periodicity equal to $(0,24)$ (when $s=$ v).

\section{Numerical Results}

We will apply the new produced method to the approximate solution for coupled differential equations of the Schrödinger type.

4.1. Error Estimation. For the numerical solution of the previously referred problem we will use variable step methods. An algorithm is called variable step when it is based on the change of the step size of the integration using a local truncation error estimation (LTEE) procedure. In the past decades several methods of constant or variable steps have been developed for the approximation of coupled differential equations of the Schrödinger type and related problems (see, e.g., [1-55]).

In our numerical tests we use local estimation procedure which is based on an embedded pair and on the fact that we have better approximation for the problems with oscillatory and/or periodical solutions having maximal algebraic order of the methods.

The local truncation error in $y_{n+1}^{L}$ is estimated by

$$
\mathrm{LTE}=\left|y_{n+1}^{H}-y_{n+1}^{L}\right|,
$$

where $y_{n+1}^{L}$ gives the lower algebraic order solution which is obtained using the twelfth-algebraic order method developed in [54] and $y_{n+1}^{H}$ gives the higher order solution which is obtained using the five-stage symmetric two-step method of fourteenth algebraic order with vanished phase-lag and its first, second, and third derivatives developed in Section 3.

In our numerical tests the changes of the step sizes are reduced on duplication of step sizes. We use the following procedure:

(i) If LTE < acc then the step size is duplicated; that is, $h_{n+1}=2 h_{n}$.

(ii) If acc $\leq$ LTE $\leq 100$ acc then the step size remains stable; that is, $h_{n+1}=h_{n}$.

(iii) If 100 acc < LTE then the step size is halved and the step is repeated; that is, $h_{n+1}=(1 / 2) h_{n}$.

In the above, $h_{n}$ is the step length used for the $n$th step of the integration and acc is the requested accuracy of the local truncation error (LTE).

Remark 12. The local extrapolation technique is also used; that is, while for a local truncation error estimation less than acc we use the lower algebraic order solution $y_{n+1}^{L}$ it is the higher algebraic order solution $y_{n+1}^{H}$ which is accepted at each point as integration.

4.2. Coupled Differential Equations. We will present the numerical solution of the coupled Schnrödinger equations 
(1) using the boundary conditions (2) and (3). Mathematical models of this form are observed in many real problems in quantum chemistry, material science, theoretical physics, atomic physics, physical chemistry and chemical physics, and so forth. The methodology fully described in [1] will be used for the approximate solution for this problem.

Equation (1) contains the potential $V$ which can be presented as (see for details [1])

$$
\begin{aligned}
V\left(x, \widehat{\mathbf{k}}_{j^{\prime} j} \widehat{\mathbf{k}}_{j j}\right)= & V_{0}(x) P_{0}\left(\widehat{\mathbf{k}}_{j^{\prime} j} \widehat{\mathbf{k}}_{j j}\right) \\
& +V_{2}(x) P_{2}\left(\widehat{\mathbf{k}}_{j^{\prime} j} \widehat{\mathbf{k}}_{j j}\right) .
\end{aligned}
$$

Based on (32), the coupling matrix element is given by

$$
\begin{aligned}
\left\langle j^{\prime} l^{\prime} ; J|V| j^{\prime \prime} l^{\prime \prime} ; J\right\rangle= & \delta_{j^{\prime} j^{\prime \prime}} \delta_{l^{\prime} l^{\prime \prime}} V_{0}(x) \\
& +f_{2}\left(j^{\prime} l^{\prime}, j^{\prime \prime} l^{\prime \prime} ; J\right) V_{2}(x),
\end{aligned}
$$

where $f_{2}$ coefficients are obtained from formulas given by Bernstein et al. [56] and $\widehat{\mathbf{k}}_{j^{\prime} j}$ is a unit vector parallel to the wave vector $\mathbf{k}_{j^{\prime} j}$ and $P_{i}, i=0,2$, are Legendre polynomials (see for details [57]). The above leads to the new form of boundary conditions:

$$
\begin{gathered}
y_{j^{\prime} l^{\prime}}^{J j l}(x)=0 \quad \text { at } x=0 \\
y_{j^{\prime} l^{\prime}}^{J j l}(x) \sim \delta_{j j^{\prime}} \delta_{l l^{\prime}} \exp \left[-i\left(k_{j j} x-\frac{1}{2} l \pi\right)\right], \\
-\left(\frac{k_{i}}{k_{j}}\right)^{1 / 2} S^{J}\left(j l ; j^{\prime} l^{\prime}\right) \exp \left[i\left(k_{j^{\prime} j} x-\frac{1}{2} l^{\prime} \pi\right)\right],
\end{gathered}
$$

where

$$
\mathbf{S}=(\mathbf{I}+\mathbf{i K})(\mathbf{I}-\mathbf{i K})^{-1}
$$

We will use the variable step method described in Section 4.1 in order to compute the cross sections for rotational excitation of molecular hydrogen by impact of various heavy particles.

We use the following parameters in our example:

$$
\begin{aligned}
\frac{2 \mu}{\hbar^{2}} & =1000.0, \\
\frac{\mu}{I} & =2.351, \\
E & =1.1, \\
V_{0}(x) & =\frac{1}{x^{12}}-2 \frac{1}{x^{6}}, \\
V_{2}(x) & =0.2283 V_{0}(x) .
\end{aligned}
$$

\begin{tabular}{|c|c|c|c|c|}
\hline Method & $N$ & $h_{\max }$ & RTC & MErr \\
\hline \multirow{3}{*}{ Method I } & 4 & 0.014 & 3.25 & $1.2 \times 10^{-3}$ \\
\hline & 9 & 0.014 & 23.51 & $5.7 \times 10^{-2}$ \\
\hline & 16 & 0.014 & 99.15 & $6.8 \times 10^{-1}$ \\
\hline \multirow{3}{*}{ Method II } & 4 & 0.056 & 1.55 & $8.9 \times 10^{-4}$ \\
\hline & 9 & 0.056 & 8.43 & $7.4 \times 10^{-3}$ \\
\hline & 16 & 0.056 & 43.32 & $8.6 \times 10^{-2}$ \\
\hline \multirow{3}{*}{ Method III } & 4 & 0.007 & 45.15 & $9.0 \times 10^{0}$ \\
\hline & 9 & & & \\
\hline & 16 & & & \\
\hline \multirow{3}{*}{ Method IV } & 4 & 0.112 & 0.39 & $1.1 \times 10^{-5}$ \\
\hline & 9 & 0.112 & 3.48 & $2.8 \times 10^{-4}$ \\
\hline & 16 & 0.112 & 19.31 & $1.3 \times 10^{-3}$ \\
\hline \multirow{3}{*}{ Method V } & 4 & 0.448 & 0.14 & $3.4 \times 10^{-7}$ \\
\hline & 9 & 0.448 & 1.37 & $5.8 \times 10^{-7}$ \\
\hline & 16 & 0.448 & 9.58 & $8.2 \times 10^{-7}$ \\
\hline \multirow{3}{*}{ Method VI } & 4 & 0.448 & 0.09 & $2.9 \times 10^{-7}$ \\
\hline & 9 & 0.448 & 1.10 & $4.5 \times 10^{-7}$ \\
\hline & 16 & 0.448 & 8.57 & $7.4 \times 10^{-7}$ \\
\hline \multirow{3}{*}{ Method VII } & 4 & 0.448 & 0.06 & $1.3 \times 10^{-7}$ \\
\hline & 9 & 0.448 & 1.04 & $1.7 \times 10^{-7}$ \\
\hline & 16 & 0.448 & 7.58 & $2.9 \times 10^{-7}$ \\
\hline \multirow{3}{*}{ Method VIII } & 4 & 0.448 & 0.04 & $8.8 \times 10^{-8}$ \\
\hline & 9 & 0.448 & 1.02 & $9.2 \times 10^{-8}$ \\
\hline & 16 & 0.448 & 7.48 & $8.9 \times 10^{-8}$ \\
\hline \multirow{3}{*}{ Method IX } & 4 & 0.448 & 0.04 & $9.7 \times 10^{-8}$ \\
\hline & 9 & 0.448 & 1.01 & $1.2 \times 10^{-7}$ \\
\hline & 16 & 0.448 & 7.15 & $2.3 \times 10^{-7}$ \\
\hline \multirow{3}{*}{ Method X } & 4 & 0.896 & 0.02 & $7.0 \times 10^{-8}$ \\
\hline & 9 & 0.896 & 0.45 & $5.7 \times 10^{-8}$ \\
\hline & 16 & 0.896 & 6.11 & $6.5 \times 10^{-8}$ \\
\hline
\end{tabular}

For our test we take $J=6$ and we will consider excitation of the rotator from $j=0$ state to levels up to $j^{\prime}=2,4$, and 6 which is equivalent to sets of four, nine, and sixteen coupled differential equations, respectively. Using the methodology fully described by Bernstein [57] and Allison [1], we consider
TABLE 1: Coupled differential equations. Real time of computation (in seconds) (RTC) and maximum absolute error (MErr) to calculate $|S|^{2}$ for the variable step methods, Method I-Method X. acc $=10^{-6}$. We note that $h_{\max }$ is the maximum step size. $N$ indicates the number of equations of the set of coupled differential equations.

the potential infinite for $x<x_{0}$. The wave functions then tend to zero and then the boundary conditions (34) are given by

$$
y_{j^{\prime} l^{\prime}}^{J j l}\left(x_{0}\right)=0
$$

For comparison purposes the following variable step methods are used:

(i) Method I. The iterative Numerov method of Allison [1].

(ii) Method II. The variable step method of Raptis and Cash [47].

(iii) Method III. The embedded Runge-Kutta Dormand and Prince method 5(4) [49].

(iv) Method IV. The embedded Runge-Kutta method ERK4(2) developed in Simos [41]. 
(v) Method V. The embedded symmetric two-step method developed in [50].

(vi) Method VI. The embedded symmetric two-step method developed in [52].

(vii) Method VII. The embedded symmetric two-step method developed in [52].

(viii) Method VIII. The embedded symmetric two-step method developed in [53].

(ix) Method IX. The embedded symmetric two-step method developed in [54].

(x) Method X. The developed embedded symmetric twostep method developed in this paper.

The results are presented in Table 1 . More specifically, we present (1) the requested real time of computation by the variable step methods mentioned above in order to calculate the square of the modulus of $\mathbf{S}$ matrix for sets of 4,9 , and 6 coupled differential equations and (2) the maximum error in the computation of the square of the modulus of $\mathbf{S}$ matrix.

\section{Conclusions}

In this paper we introduce, for the first time in the literature, a new five-stage symmetric two-step fourteenth-algebraic order family of methods and we produced a method of the family with vanished phase-lag and its first, second, and third derivatives. In this paper,

(1) we introduced the new family of methods,

(2) we developed the new method of the new family with vanished phase-lag and its first, second, and third derivatives,

(3) we analyzed the local truncation error,

(4) we analyzed the interval of periodicity and the stability of the new developed method,

(5) finally, we analyzed the efficiency of the new obtained method on the numerical solution for coupled Schrödinger equations.

From the analysis presented above, we conclude that the new developed method is much more efficient than the known ones for the numerical solution for the coupled Schrödinger equations.

All computations were carried out on IBM PC-AT compatible 80486 using double precision arithmetic with 16 significant digits' accuracy (IEEE standard).

\section{Appendix}

A. Formulae of $T_{j}, j=0(1) 3$, and $T_{\text {denom }}$

$$
\begin{aligned}
& T_{0}=\left(45469 v^{8} b_{2}+7038360 b_{2} v^{6}+652886640 b_{2} v^{4}-265712832000\right. \\
& \left.+\left(-265712832000 b_{1}-132856416000 b_{2}\right) v^{2}\right) \cos (v)-45469 v^{8} b_{2} \\
& +431033400 b_{2} v^{6}-33866990640 b_{2} v^{4}+\left(-132856416000 b_{0}-132856416000 b_{2}\right) v^{2} \\
& -132856416000 a_{4} \text {, } \\
& T_{1}=-2067429961\left(-\frac{265712832000}{45469}+v^{8} b_{2}+\frac{7038360 b_{2} v^{6}}{45469}+\frac{652886640 b_{2} v^{4}}{45469}\right. \\
& \left.+\left(-\frac{265712832000 b_{1}}{45469}-\frac{132856416000 b_{2}}{45469}\right) v^{2}\right)^{2} \sin (v)-39837369710880 \\
& \cdot\left(v^{12} b_{2}{ }^{2}-\frac{4393400 v^{10} b_{2}{ }^{2}}{28973}-\frac{26360400 v^{8} b_{2}}{28973}\left(b_{0}+2 b_{1}+\frac{153226717033 b_{2}}{4994087615}\right)\right. \\
& -\frac{17669903328000 b_{2} v^{6}}{188196191}\left(b_{0}+\frac{45469 a_{4}}{3519180}-\frac{16290 b_{1}}{133}-\frac{8012 b_{2}}{133}+\frac{45469}{1759590}\right) \\
& -\frac{819540618336000 b_{2} v^{4}}{188196191}\left(b_{0}+\frac{8379 a_{4}}{259082}+\frac{13439282 b_{1}}{129541}+\frac{6849182 b_{2}}{129541}-\frac{513135}{129541}\right)
\end{aligned}
$$




$$
\begin{aligned}
& -\frac{1639081236672000 v^{2} b_{2}}{188196191}\left(a_{4}+\frac{13439282}{129541}\right) \\
& +\left(\frac{166768965158400000 a_{4}}{188196191}-\frac{333537930316800000}{188196191}\right) b_{2} \\
& \left.+\frac{333537930316800000 a_{4} b_{1}}{188196191}-\frac{333537930316800000 b_{0}}{188196191}\right) v, \\
& T_{2}=-94003972896709\left(-\frac{265712832000}{45469}+v^{8} b_{2}+\frac{7038360 b_{2} v^{6}}{45469}+\frac{652886640 b_{2} v^{4}}{45469}\right. \\
& \left.+\left(-\frac{265712832000 b_{1}}{45469}-\frac{132856416000 b_{2}}{45469}\right) v^{2}\right)^{3} \cos (v)+5434096090152008160 v^{20} b_{2}^{3} \\
& -1653746424225668236800 v^{18} b_{2}{ }^{3}-11536196067878150592000 b_{2}^{2} \\
& \cdot\left(b_{0}+2 b_{1}+\frac{227930737228 b_{2}}{4994087615}\right) v^{16}-2295953942267723189760000 b_{2}^{2} \\
& \cdot\left(b_{0}+\frac{45469 a_{4}}{5278770}-\frac{16290 b_{1}}{133}-\frac{7009130426558198 b_{2}}{125536380378255}+\frac{45469}{2639385}\right) v^{14} \\
& -194734009025384053800960000 b_{2}^{2}\left(b_{0}+\frac{6730730601 a_{4}}{290822990464}+\frac{379717697333 b_{1}}{18176436904}\right. \\
& \left.+\frac{127130652893979671 b_{2}}{15971271578806720}-\frac{356864310411}{145411495232}\right) v^{12} \\
& -48153927914830003875840000000 b_{2}\left(\frac{541122054664 b_{2}{ }^{2}}{24970438075}\right. \\
& +\left(\frac{72734780969 b_{0}}{99881752300}+\frac{12770448547 a_{4}}{1198581027600}+\frac{2191635189987 b_{1}}{49940876150}+\frac{63965810599}{599290513800}\right) \\
& \left.\cdot b_{2}+b_{1}\left(b_{0}+2 b_{1}\right)\right) v^{10}-2981589039377359573155840000000 b_{2} \\
& \cdot\left(-\frac{1773970067669 b_{2}^{2}}{73624597200}+\left(\frac{90405467881 b_{0}}{147249194400}+\frac{58393539227 a_{4}}{6184466164800}\right.\right. \\
& \left.-\frac{8073535624019 b_{1}}{73624597200}+\frac{141363876421}{114527151200}\right) b_{2}-\frac{16290 b_{1}^{2}}{133}+\left(b_{0}-\frac{45469 a_{4}}{14076720}+\frac{591097}{7038360}\right) b_{1} \\
& \left.+\frac{45469 b_{0}}{1005480}\right) v^{8}-46095957244299145061007360000000 b_{2}\left(\frac{3424591 b_{2}^{2}}{129541}\right. \\
& +\left(\frac{1}{2} b_{0}-\frac{12668968199 a_{4}}{682950515280}+\frac{13568823 b_{1}}{129541}-\frac{2869992664019}{341475257640}\right) b_{2}+\frac{13439282 b_{1}^{2}}{129541} \\
& \left.+\left(b_{0}-\frac{11172 a_{4}}{129541}-\frac{2907765}{129541}\right) b_{1}+\frac{69825 b_{0}}{259082}+\frac{45469 a_{4}}{23317380}+\frac{45469}{11658690}\right) v^{6}
\end{aligned}
$$




$$
\begin{aligned}
& -553151486931589740732088320000000 b_{2}\left(\left(-\frac{3}{8} a_{4}+\frac{7237805}{518164}\right) b_{2}+\left(-\frac{3}{4} a_{4}+\frac{6719641}{259082}\right) b_{1}\right. \\
& \left.+b_{0}+\frac{13965 a_{4}}{1036328}-\frac{855225}{518164}\right) v^{4}+\left(\left(-14070153905048863102795776000000000 a_{4}\right.\right. \\
& +28140307810097726205591552000000000) b_{2}{ }^{2}+\left(\left(-56280615620195452411183104000000000 a_{4}\right.\right. \\
& +56280615620195452411183104000000000) b_{1}+28140307810097726205591552000000000 b_{0} \\
& \left.-276575743465794870366044160000000 a_{4}-28693459297029315946323640320000000\right) b_{2} \\
& \left.+56280615620195452411183104000000000 b_{1}\left(-a_{4} b_{1}+b_{0}\right)\right) v^{2} \\
& +\left(9380102603365908735197184000000000 a_{4}-18760205206731817470394368000000000\right) b_{2} \\
& +18760205206731817470394368000000000 a_{4} b_{1}-18760205206731817470394368000000000 b_{0}, \\
& T_{3}=4274266643640461521\left(-\frac{265712832000}{45469}+v^{8} b_{2}+\frac{7038360 b_{2} v^{6}}{45469}+\frac{652886640 b_{2} v^{4}}{45469}\right. \\
& \left.+\left(-\frac{265712832000 b_{1}}{45469}-\frac{132856416000 b_{2}}{45469}\right) v^{2}\right)^{4} \sin (v)-988331660492486636108160 \\
& \cdot\left(v^{26} b_{2}{ }^{4}-\frac{703331165780 v^{24} b_{2}{ }^{4}}{1317373337}-\frac{17573600 b_{2}{ }^{3} v^{22}}{4139}\left(b_{0}+2 b_{1}+\frac{52341891953 b_{2}}{998817523}\right)\right. \\
& -\frac{229708743264000 v^{20} b_{2}^{3}}{188196191}\left(b_{0}+\frac{45469 a_{4}}{6099912}-\frac{16290 b_{1}}{133}-\frac{20280808515977707 b_{2}}{362660654426070}+\frac{45469}{3049956}\right) \\
& -\frac{1218751529857909344000 v^{18} b_{2}^{3}}{8557092608579}\left(b_{0}+\frac{8254669605 a_{4}}{385284849578}-\frac{663166829662 b_{1}}{192642424789}\right. \\
& \left.-\frac{2052773129691092548765 b_{2}}{192414629552462777647}-\frac{394861571517}{192642424789}\right) \\
& -\frac{11673827561088000000 b_{2}^{2} v^{16}}{188196191}\left(\frac{25981841558644066102303 b_{2}^{2}}{997636444251855529000}\right. \\
& +\left(\frac{14436736503248 b_{0}}{20643288160585}+\frac{672095448881 a_{4}}{83900671932000}+\frac{61372103581289784 b_{1}}{1135380848832175}-\frac{6194383101859}{41950335966000}\right) b_{2} \\
& \left.+b_{1}\left(b_{0}+2 b_{1}\right)\right)-\frac{84512160980083888128000000 b_{2}^{2} v^{14}}{8557092608579}\left(-\frac{200253323882994581 b_{2}^{2}}{15064365645390600}\right.
\end{aligned}
$$




$$
\begin{aligned}
& +\left(\frac{17737276339168999 b_{0}}{30128731290781200}+\frac{4562567732722819 a_{4}}{632703357106405200}-\frac{56067458474270819 b_{1}}{792861349757400}+\frac{170062002887713063}{316351678553202600}\right) b_{2} \\
& \left.-\frac{105220 b_{1}^{2}}{1197}+\left(b_{0}+\frac{45469 a_{4}}{12669048}+\frac{591097}{15836310}\right) b_{1}+\frac{45469 b_{0}}{3016440}\right) \\
& -\frac{244441255786320657705467904000000 b_{2}^{2} v^{12}}{389082443819478551}\left(-\frac{18787681229658547 b_{2}^{2}}{7985635789403360}\right. \\
& +\left(\frac{8969884197456053 b_{0}}{15971271578806720}+\frac{3422548232166571 a_{4}}{383310517891361280}-\frac{136160476648092927 b_{1}}{7985635789403360}-\frac{280627602486327149}{191655258945680640}\right) b_{2} \\
& -\frac{222153379571 b_{1}^{2}}{9088218452}+\left(b_{0}+\frac{380984751 a_{4}}{72705747616}-\frac{154401947847}{36352873808}\right) b_{1} \\
& \left.+\frac{1650933921 b_{0}}{36352873808}+\frac{2067429961 a_{4}}{8724689713920}+\frac{2067429961}{4362344856960}\right)-\frac{443126540219477925888000000000 b_{2} v^{10}}{8557092608579} \\
& \cdot\left(\frac{4028587739494373897 b_{2}^{3}}{438820490554820000}+\left(\frac{434912403523211147 b_{0}}{877640981109640000}+\frac{2926099586132929 a_{4}}{1755281962219280000}\right.\right. \\
& \left.+\frac{15313712383120673847 b_{1}}{438820490554820000}-\frac{144221396158735377}{877640981109640000}\right) b_{2}^{2}+\left(\frac{15500862502 b_{1}^{2}}{454007965}\right. \\
& +\left(\frac{72734780969 b_{0}}{49940876150}-\frac{7528278019 a_{4}}{1997635046000}-\frac{539180954813}{499408761500}\right) b_{1} \\
& \left.\left.+\frac{98566938253 b_{0}}{1997635046000}+\frac{49343 a_{4}}{87868000}-\frac{100387}{2196700}\right) b_{2}+b_{1}^{2}\left(b_{0}+2 b_{1}\right)\right) \\
& -\frac{623776823123832930890612736000000000 v^{8} b_{2}}{389082443819478551}\left(-\frac{665172562469 b_{2}{ }^{3}}{73624597200}\right. \\
& +\left(\frac{53593169281 b_{0}}{147249194400}-\frac{2554367727834151 a_{4}}{1811388909895488000}-\frac{547986492173 b_{1}}{8180510800}+\frac{267706836641709133}{143004387623328000}\right) b_{2}^{2} \\
& +\left(-\frac{5855940613619 b_{1}^{2}}{36812298600}+\left(\frac{90405467881 b_{0}}{73624597200}+\frac{2390206957 a_{4}}{309223308240}+\frac{1205462023201}{386529135300}\right) b_{1}\right. \\
& \left.\left.+\frac{28440383893 b_{0}}{220873791600}+\frac{12770448547 a_{4}}{7421359397760}-\frac{109828438403}{3710679698880}\right) b_{2}+\left(-\frac{16290 b_{1}^{2}}{133}+\left(b_{0}+\frac{45469 a_{4}}{1407672}+\frac{45469}{185220}\right) b_{1}+\frac{45469 b_{0}}{502740}\right) b_{1}\right) \\
& -\frac{1421741999377084654844751052800000000 b_{2} v^{6}}{389082443819478551}\left(\left(\frac{23281985157 a_{4}}{167808706810}-\frac{2683972235033}{83904353405}\right) b_{2}^{2}\right. \\
& +\left(\left(\frac{96906582357 a_{4}}{83904353405}-\frac{14553382412876}{83904353405}\right) b_{1}+b_{0}+\frac{58393539227 a_{4}}{1761991421505}+\frac{439542930952}{251713060215}\right) b_{2} \\
& \left.+\left(\frac{29449838880 a_{4}}{16780870681}-\frac{3607051694400}{16780870681}\right) b_{1}{ }^{2}+\left(-\frac{3995270092 a_{4}}{352398284301}+\frac{51938511196}{352398284301}\right) b_{1}+\frac{9988175230 b_{0}}{117466094767}\right) \\
& +\frac{231449118350975844469748269056000000000 b_{2} v^{4}}{389082443819478551}\left(\left(-\frac{1}{4} a_{4}+\frac{1}{2}\right) b_{2}^{2}\right. \\
& +\left(\left(-a_{4}+1\right) b_{1}+\frac{1}{2} b_{0}+\frac{12668968199 a_{4}}{2731802061120}+\frac{430638152579}{1365901030560}\right) b_{2} \\
& \left.-a_{4} b_{1}^{2}+\left(b_{0}+\frac{2793 a_{4}}{129541}+\frac{513135}{259082}\right) b_{1}-\frac{19551 b_{0}}{518164}-\frac{45469 a_{4}}{186539040}-\frac{45469}{93269520}\right)
\end{aligned}
$$




$$
\begin{aligned}
& +\left(\left(\frac{2943610198401984531229664870400000000000 a_{4}}{389082443819478551}-\frac{5887220396803969062459329740800000000000}{389082443819478551}\right) b_{2}{ }^{3}\right. \\
& +\left(\left(\frac{17661661190411907187377989222400000000000 a_{4}}{389082443819478551}-\frac{23548881587215876249837318963200000000000}{389082443819478551}\right) b_{1}\right. \\
& +\frac{66150270245146553335607721984000000000}{9489815702914111}-\frac{5887220396803969062459329740800000000000 b_{0}}{389082443819478551} \\
& \left.+\frac{173586838763231883352311201792000000000 a_{4}}{389082443819478551}\right) b_{2}^{2} \\
& +\left(\left(\frac{35323322380823814374755978444800000000000 a_{4}}{389082443819478551}-\frac{23548881587215876249837318963200000000000}{389082443819478551}\right) b_{1}^{2}\right. \\
& +\left(\frac{6002944955979456984694203875328000000000}{389082443819478551}-\frac{23548881587215876249837318963200000000000 b_{0}}{389082443819478551}\right. \\
& \left.+\frac{347173677526463766704622403584000000000 a_{4}}{389082443819478551}\right) b_{1}+\frac{382004678522076633240905318400000000000}{389082443819478551} \\
& \left.-\frac{3118884115619164654453063680000000000 a_{4}}{389082443819478551}-\frac{289311397938719805587185336320000000000 b_{0}}{389082443819478551}\right) b_{2} \\
& \left.-\frac{23548881587215876249837318963200000000000 b_{1}^{2}\left(-a_{4} b_{1}+b_{0}\right)}{389082443819478551}\right) v^{2} \\
& +\left(\frac{11774440793607938124918659481600000000000}{389082443819478551}-\frac{5887220396803969062459329740800000000000 a_{4}}{389082443819478551}\right) b_{2}{ }^{2} \\
& +\left(\left(\frac{23548881587215876249837318963200000000000}{389082443819478551}-\frac{23548881587215876249837318963200000000000 a_{4}}{389082443819478551}\right) b_{1}\right. \\
& -\frac{6002944955979456984694203875328000000000}{389082443819478551}+\frac{11774440793607938124918659481600000000000 b_{0}}{389082443819478551} \\
& \left.\left.-\frac{57862279587743961117437067264000000000 a_{4}}{389082443819478551}\right) b_{2}+\frac{23548881587215876249837318963200000000000 b_{1}\left(-a_{4} b_{1}+b_{0}\right)}{389082443819478551}\right) v \text {, } \\
& T_{\text {denom }}=45469 v^{8} b_{2}+7038360 b_{2} v^{6}+652886640 b_{2} v^{4}-265712832000+\left(-265712832000 b_{1}-132856416000 b_{2}\right) v^{2} \text {. }
\end{aligned}
$$

\section{B. Formulae for the Coefficients of the Produced Method $T_{j}, j=4(1) 7, T_{\text {denom } 1}$, and $T_{\text {denom } 2}$}

$$
\begin{aligned}
T_{4} & =\left(15277584 v^{6}+844603200 v^{4}\right. \\
& \left.+15669279360 v^{2}\right)(\cos (v))^{3}+\left(\left(-2182512 v^{7}\right.\right. \\
& -153643056 v^{5}-4378489920 v^{3}
\end{aligned}
$$

$$
\begin{aligned}
& +15669279360 v) \sin (v)+363752 v^{8} \\
& -2593839192 v^{6}+148398964560 v^{4} \\
& \left.+672116719680 v^{2}+797138496000\right)(\cos (v))^{2} \\
& +\left(\left(-4365024 v^{7}+20674325616 v^{5}\right.\right. \\
& \left.-490147842240 v^{3}-812807775360 v\right) \sin (v) \\
& -30555168 v^{6}-1689206400 v^{4}
\end{aligned}
$$




$$
\begin{aligned}
& \left.-31338558720 v^{2}\right) \cos (v)+\left(13095072 v^{7}\right. \\
& \left.+1013523840 v^{5}+31338558720 v^{3}\right) \sin (v) \\
& +727504 v^{8}-5149484424 v^{6}+206280915120 v^{4} \\
& +937829551680 v^{2}-797138496000 \\
& T_{5}=\left(-181876 v^{8}-17295684 v^{6}-1200197880 v^{4}\right. \\
& \left.+128939096160 v^{2}+398569248000\right)(\cos (v))^{2} \\
& +\left(\left(-2182512 v^{7}-176559432 v^{5}-5645394720 v^{3}\right.\right. \\
& -7834639680 v) \sin (v)-7638792 v^{6} \\
& \left.+25862004000 v^{4}-406403887680 v^{2}\right) \cos (v) \\
& +\left(-1091256 v^{7}+5180039592 v^{5}\right. \\
& \left.-161329966560 v^{3}+406403887680 v\right) \sin (v) \\
& -363752 v^{8}-38410764 v^{6}-2294820360 v^{4} \\
& +277464791520 v^{2}-398569248000 \\
& T_{6}=\left(-88570944000 v^{2}-265712832000\right)(\cos (v))^{2} \\
& -177141888000 v^{2}+265712832000 \\
& T_{\text {denom } 1}=v^{3}\left(v \left(v^{6}+\frac{1191127 v^{4}}{45469}+\frac{6478080 v^{2}}{45469}\right.\right. \\
& \left.-\frac{4570206480}{45469}\right)(\cos (v))^{2}+\left(\left(16 v^{6}\right.\right. \\
& \left.+\frac{50850600 v^{4}}{45469}+\frac{1487650080 v^{2}}{45469}-\frac{2611546560}{45469}\right) \\
& \cdot \sin (v)+8 v^{5}+\frac{1724133600 v^{3}}{45469} \\
& \left.-\frac{135467962560 v}{45469}\right) \cos (v)+\left(8 v^{6}\right. \\
& -\frac{1718677320 v^{4}}{45469}+\frac{29638785120 v^{2}}{45469} \\
& \left.+\frac{135467962560}{45469}\right) \sin (v)+2 v^{7}+\frac{12521841 v^{5}}{45469}
\end{aligned}
$$

$$
\begin{aligned}
& \left.+\frac{674562000 v^{3}}{45469}+\frac{7181753040 v}{45469}\right) \\
T_{7} & =\left(-727504 v^{5}+56306880 v^{3}+5223093120 v\right) \\
& \cdot(\cos (v))^{3}+\left(\left(727504 v^{6}+67219440 v^{4}\right.\right. \\
& \left.+2247792960 v^{2}+5223093120\right) \sin (v)-90938 v^{7} \\
& +581713426 v^{5}-48439997760 v^{3} \\
& +474137868960 v)(\cos (v))^{2}+\left(\left(1455008 v^{6}\right.\right. \\
& -6907446960 v^{4}+211658354880 v^{2} \\
& -270935925120) \sin (v)+1455008 v^{5} \\
& \left.-112613760 v^{3}-10446186240 v\right) \cos (v) \\
& +\left(-4365024 v^{6}-337841280 v^{4}\right. \\
& \left.-10446186240 v^{2}\right) \sin (v)-181876 v^{7} \\
+ & -1141692670 v^{5}-22742250720 v^{3} \\
& -203201943840 v,
\end{aligned}
$$$$
T_{\text {denom } 2}=\left(45469 v^{7}+1191127 v^{5}+6478080 v^{3}\right.
$$$$
-4570206480 v)(\cos (v))^{2}+\left(\left(727504 v^{6}\right.\right.
$$$$
\left.+50850600 v^{4}+1487650080 v^{2}-2611546560\right)
$$$$
\cdot \sin (v)+363752 v^{5}+1724133600 v^{3}
$$$$
-135467962560 v) \cos (v)+\left(363752 v^{6}\right.
$$$$
-1718677320 v^{4}+29638785120 v^{2}
$$$$
+135467962560) \sin (v)+90938 v^{7}+12521841 v^{5}
$$$$
+674562000 v^{3}+7181753040 v .
$$

\section{Formulae of the Asymptotic Form of the Local Truncation Errors} $\mathrm{LTE}_{\mathrm{CL}}=\mathrm{LTE}_{\mathrm{NM} 2 \mathrm{~S} 5 \mathrm{~S} 3 \mathrm{DV}}=a_{0}$

Formulae of the asymptotic form of the local truncation errors are as follows: 
$\mathrm{LTE}_{\mathrm{CL}}$

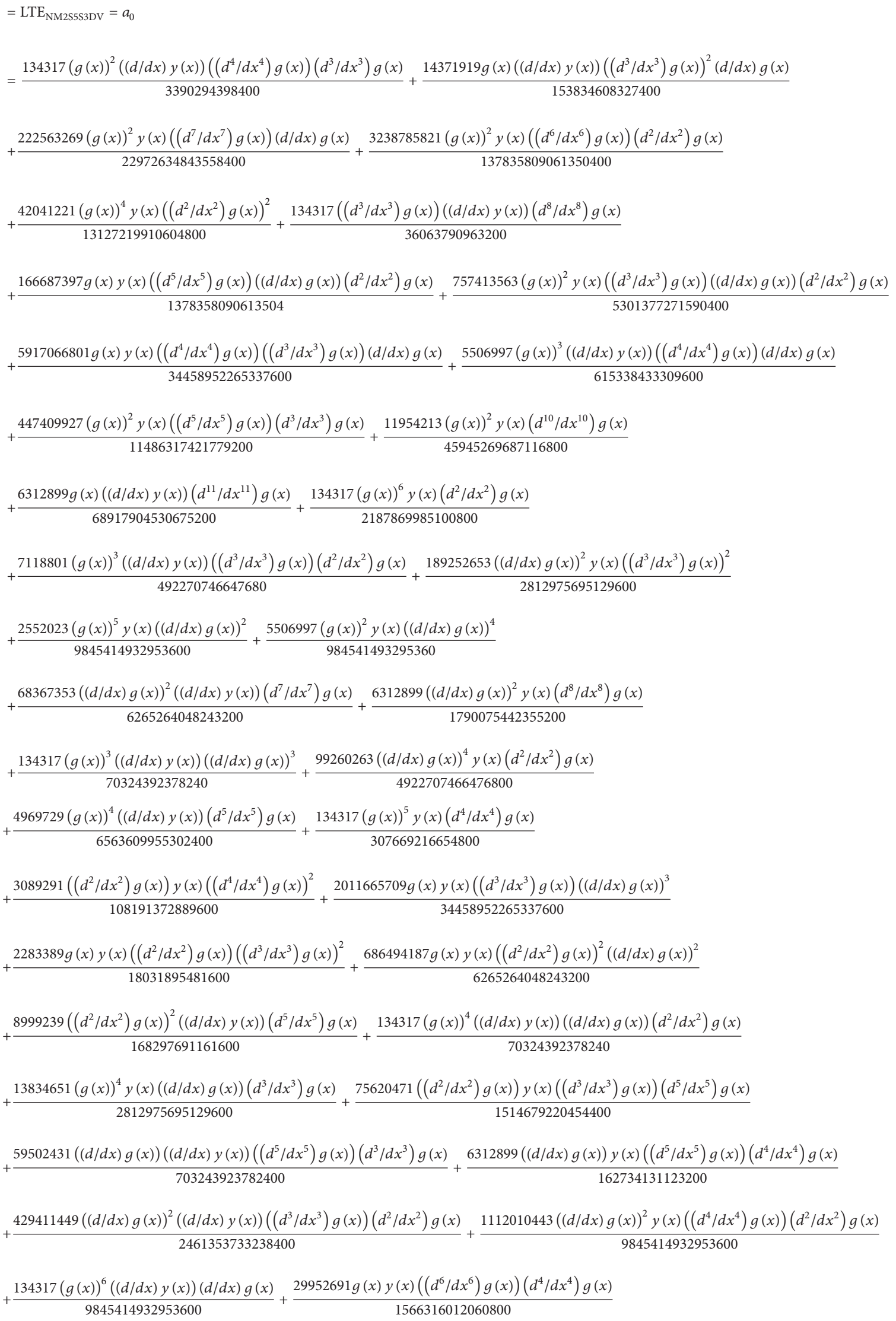




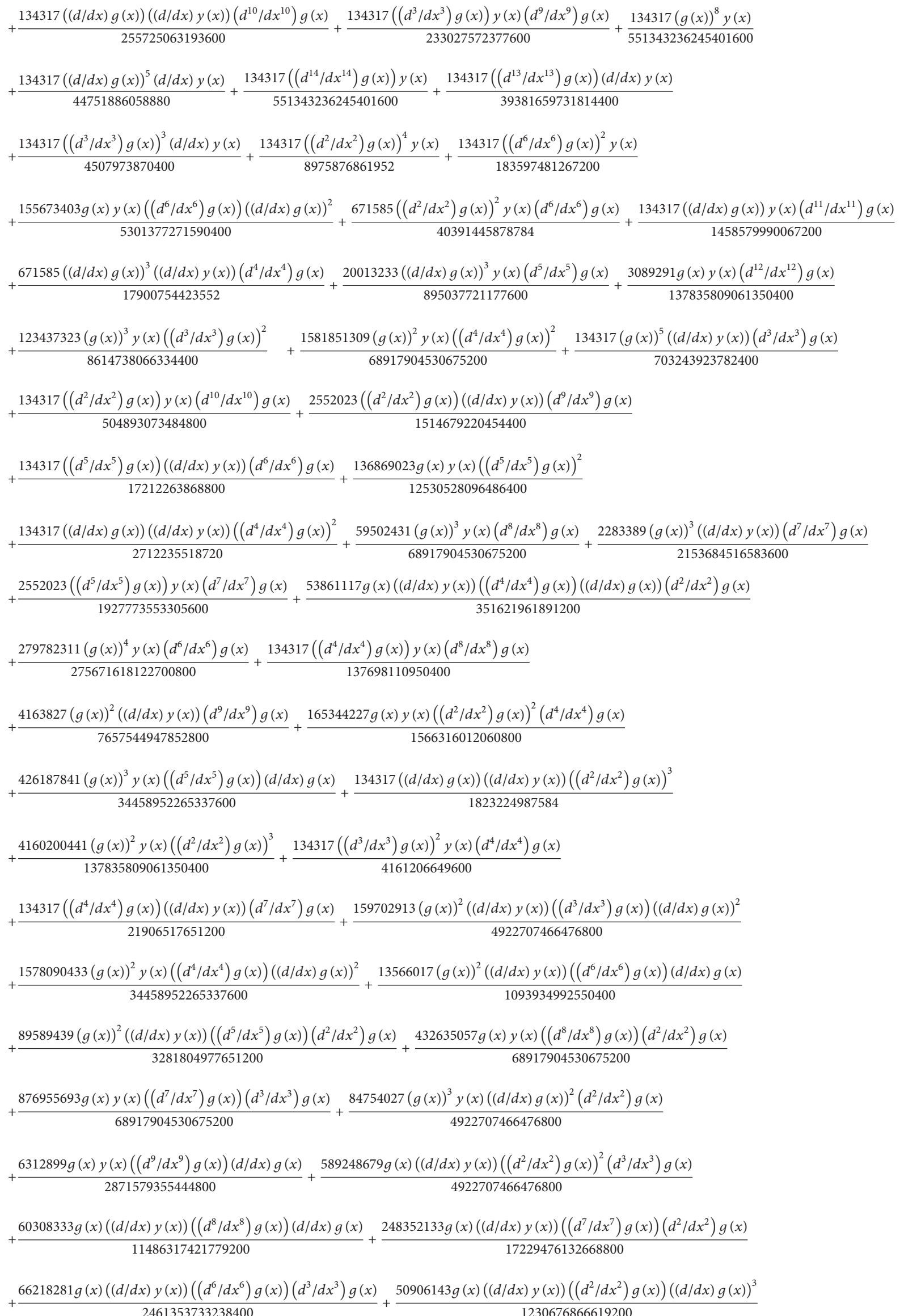




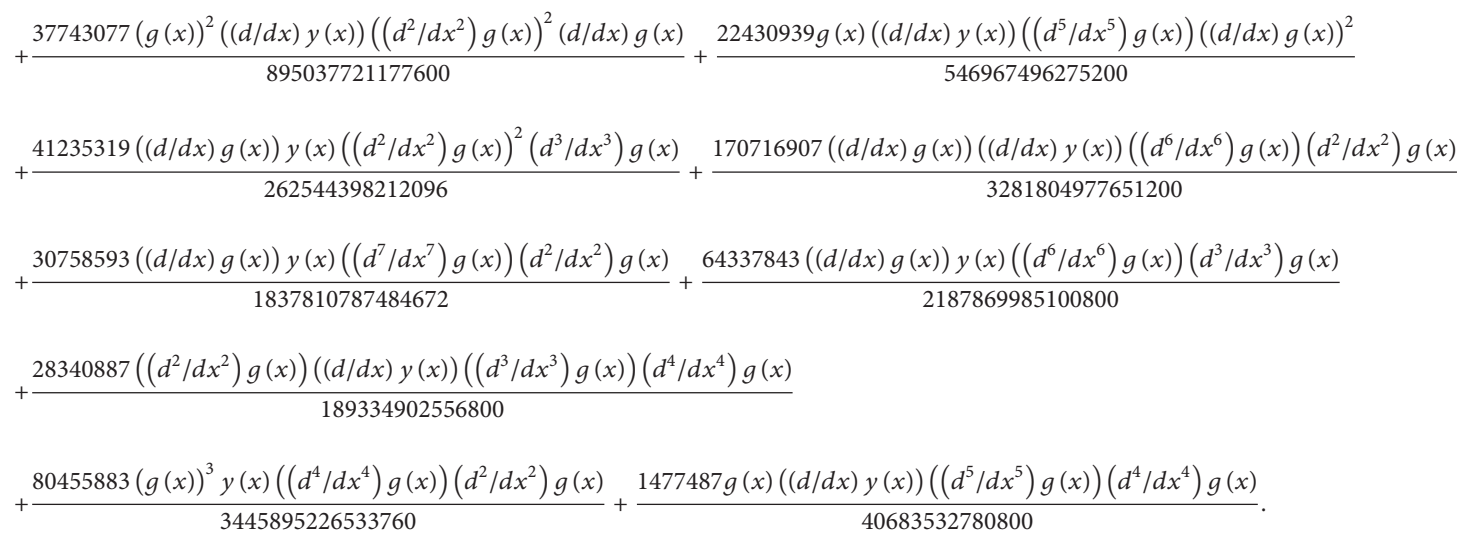

\section{Formulae for the Stability Polynomials} $T_{k}, k=8,9$, and $T_{\mathrm{denom} 3}$

$$
\begin{aligned}
T_{8} & =159532850484000 v^{2} s^{2} \\
& +26588808414000 s^{4} v^{2} \\
& +285508033854 s^{6} v^{2} \\
& -30236885 s^{8} v^{2}+1881961910 s^{6} v^{4} \\
& -7406900100 s^{2} v^{8} \\
& -766584691740 s^{2} v^{6} \\
& -47158235397000 s^{2} v^{4} \\
& +17776560240 \sin (v) v^{9} \\
& +17776560240 \cos (v) v^{8} \\
& -83991760628400 \sin (v) v^{7} \\
& +84258409032000 \cos (v) v^{6} \\
& +1448447428814400 \sin (v) v^{5} \\
& -6620319330307200 \cos (v) v^{4} \\
& +6620319330307200 \sin (v) v^{3} \\
& +33124136824800 v^{6} \\
& +239299275726000 v^{4} \\
& +641047557915 v^{8} \\
& -17776560240 \sin (v) s^{2} v^{7} \\
- & 124435921680 \cos (v) s^{2} v^{6}
\end{aligned}
$$

$$
\begin{aligned}
& +84382844953680 \sin (v) s^{2} v^{5} \\
& +421292045160000 \cos (v) s^{2} v^{4} \\
& -2628065155262400 \sin (v) s^{2} v^{3} \\
& -6620319330307200 \cos (v) s^{2} v^{2} \\
& +6620319330307200 \sin (v) s^{2} v \\
& -15953285048400 s^{4} \\
& -171982326600 s^{6} \\
& +5555175075 v^{10} \\
& +18142131 s^{8} \\
& +1111035015 v^{10} \cos (2 v) \\
& -18142131 s^{8} \cos (2 v) \\
& +29105188245 v^{8} \cos (2 v) \\
& +171982326600 s^{6} \cos (2 v) \\
& +158291884800 v^{6} \cos (2 v) \\
& +15953285048400 s^{4} \cos (2 v) \\
& -111672995338800 v^{4} \cos (2 v) \\
& +17776560240 v^{9} \sin (2 v) \\
& +1242534411000 v^{7} \sin (2 v) \\
& +36350729704800 v^{5} \sin (2 v) \\
& -63813140193600 v^{3} \sin (2 v) \\
& -17776560240 s^{2} v^{7} \sin (2 v) \\
& -1438076573640 s^{2} v^{5} \sin (2 v)
\end{aligned}
$$




$$
\begin{aligned}
& -45981739994400 s^{2} v^{3} \sin (2 v) \\
& -63813140193600 s^{2} v \sin (2 v) \\
& -6047377 s^{8} v^{2} \cos (2 v) \\
& +376392382 s^{6} v^{4} \cos (2 v) \\
& -1481380020 s^{2} v^{8} \cos (2 v) \\
& +58456619346 s^{6} v^{2} \cos (2 v) \\
& -140873346180 s^{2} v^{6} \cos (2 v) \\
& +5317761682800 s^{4} v^{2} \cos (2 v) \\
& -9775611732600 s^{2} v^{4} \cos (2 v) \\
& -31906570096800 v^{2} s^{2} \cos (2 v), \\
& T_{9}=9930478995460800 v^{2} s^{2} \\
& -2779874954539200 s^{4} v^{2} \\
& -571016067708 s^{6} v^{2} \\
& +60473770 s^{8} v^{2} \\
& -3763923820 s^{6} v^{4} \\
& +14813800200 s^{2} v^{8} \\
& -105011921485800 s^{2} v^{6} \\
& +4569025673646000 s^{2} v^{4} \\
& -204430442760 \sin (v) v^{9} \\
& +44441400600 \cos (v) v^{8} \\
& -15689049845400 \sin (v) v^{7} \\
& -3439646532000 \cos (v) v^{6} \\
& -483042711060000 \sin (v) v^{5} \\
& -319065700968000 \cos (v) v^{4} \\
& +63813140193600 \sin (v) v^{3} \\
& -2295045137952000 v^{6} \\
& +1655079832576800 v^{4} \\
& +70008688347210 v^{8} \\
& +204430442760 \sin (v) s^{2} v^{7} \\
& -311089804200 \cos (v) s^{2} v^{6} \\
& +15884592008040 \sin (v) s^{2} v^{5}
\end{aligned}
$$

$$
\begin{aligned}
& -17198232660000 \cos (v) s^{2} v^{4} \\
& +492673721349600 \sin (v) s^{2} v^{3} \\
& -319065700968000 \cos (v) s^{2} v^{2} \\
& +63813140193600 \sin (v) s^{2} v \\
& +1655079832576800 s^{4} \\
& +343964653200 s^{6} \\
& -11110350150 v^{10} \\
& -36284262 s^{8} \\
& -2222070030 v^{10} \cos (2 v) \\
& +36284262 s^{8} \cos (2 v) \\
& +14214167564310 v^{8} \cos (2 v) \\
& -343964653200 s^{6} \cos (2 v) \\
& -1183631345265600 v^{6} \cos (2 v) \\
& -1655079832576800 s^{4} \cos (2 v) \\
& +11585558828037600 v^{4} \cos (2 v) \\
& +35553120480 v^{9} \sin (2 v) \\
& -168783466467600 v^{7} \sin (2 v) \\
& +5171871901492800 v^{5} \sin (2 v) \\
& -6620319330307200 v^{3} \sin (2 v) \\
& -35553120480 s^{2} v^{7} \sin (2 v) \\
& +168392382142320 s^{2} v^{5} \sin (2 v) \\
& -3992254175044800 s^{2} v^{3} \sin (2 v) \\
& -6620319330307200 s^{2} v \sin (2 v) \\
& +12094754 s^{8} v^{2} \cos (2 v) \\
& -752784764 s^{6} v^{4} \cos (2 v) \\
& +2962760040 s^{2} v^{8} \cos (2 v) \\
& -116913238692 s^{6} v^{2} \cos (2 v) \\
& -21126820218840 s^{2} v^{6} \cos (2 v) \\
& -530284710614400 s^{4} v^{2} \cos (2 v) \\
& +1208709566341200 s^{2} v^{4} \cos (2 v) \\
& +3310159665153600 v^{2} s^{2} \cos (2 v)
\end{aligned}
$$




$$
\begin{aligned}
& +35680944852000 s^{4} v^{4} \\
& +3439646532000 s^{2} v^{4} \cos (3 v) \\
& +63813140193600 v^{2} s^{2} \cos (3 v) \\
& +63813140193600 v^{3} \sin (3 v) \\
& +821253508200 v^{7} \sin (3 v) \\
& +27462410488800 v^{5} \sin (3 v) \\
& +8888280120 v^{9} \sin (3 v) \\
& +63813140193600 v^{4} \cos (3 v) \\
& -8888280120 v^{8} \cos (3 v) \\
& +687929306400 v^{6} \cos (3 v) \\
& -8888280120 s^{2} v^{7} \sin (3 v) \\
& +62217960840 s^{2} v^{6} \cos (3 v) \\
& +7136188970400 s^{4} v^{4} \cos (2 v) \\
& -625711345560 s^{2} v^{5} \sin (3 v) \\
& -17831400199200 s^{2} v^{3} \sin (3 v) \\
& +63813140193600 s^{2} v \sin (3 v), \\
& T_{\text {denom3 }} \\
& =1111035015 v^{10} \cos (2 v) \\
& +17776560240 \sin (v) v^{9} \\
& +17776560240 v^{9} \sin (2 v) \\
& +5555175075 v^{10} \\
& +17776560240 \cos (v) v^{8} \\
& +29105188245 v^{8} \cos (2 v) \\
& -83991760628400 \sin (v) v^{7} \\
& +1242534411000 v^{7} \sin (2 v) \\
& +641047557915 v^{8} \\
& +84258409032000 \cos (v) v^{6} \\
& +158291884800 v^{6} \cos (2 v) \\
& +1448447428814400 \sin (v) v^{5} \\
& +36350729704800 v^{5} \sin (2 v) \\
& +33124136824800 v^{6}
\end{aligned}
$$

\section{Additional Points}

Theodore E. Simos is highly cited researcher, active member of the European Academy of Sciences and Arts, active member of the European Academy of Sciences, and corresponding member of European Academy of Arts, Sciences, and Humanities.

\section{Competing Interests}

The authors declare that they have no competing interests.

\section{References}

[1] A. C. Allison, "The numerical solution of coupled differential equations arising from the Schrödinger equation," Journal of Computational Physics, vol. 6, no. 3, pp. 378-391, 1970.

[2] J. D. Lambert and I. A. Watson, "Symmetric multistep methods for periodic initial values problems," Journal of the Institute of Mathematics and Its Applications, vol. 18, no. 2, pp. 189-202, 1976.

[3] T. E. Simos and P. S. Williams, "A finite-difference method for the numerical solution of the Schrödinger equation," Journal of Computational and Applied Mathematics, vol. 79, no. 2, pp. 189205, 1997.

[4] R. M. Thomas, "Phase properties of high order, almost $P$-stable formulae," BIT Numerical Mathematics, vol. 24, no. 2, pp. 225$238,1984$.

[5] A. D. Raptis and T. E. Simos, "A four-step phase-fitted method for the numerical integration of second order initial value problems," BIT Numerical Mathematics, vol. 31, no. 1, pp. 160$168,1991$.

[6] Z. A. Anastassi and T. E. Simos, "A parametric symmetric linear four-step method for the efficient integration of the Schrödinger equation and related oscillatory problems," Journal of Computational and Applied Mathematics, vol. 236, no. 16, pp. 3880-3889, 2012.

[7] Z. A. Anastassi and T. E. Simos, "An optimized Runge-Kutta method for the solution of orbital problems," Journal of Computational and Applied Mathematics, vol. 175, no. 1, pp. 1-9, 2005.

[8] P. Atkins and R. Friedman, Molecular Quantum Mechanics, Oxford University Press, Oxford, UK, 2011.

[9] M. M. Chawla and P. S. Rao, "A Noumerov-type method with minimal phase-lag for the integration of second order periodic initial-value problems. II: explicit method," Journal of Computational and Applied Mathematics, vol. 15, no. 3, pp. 329337, 1986.

[10] M. M. Chawla and P. S. Rao, "An explicit sixth-order method with phase-lag of order eight for $y^{\prime \prime}=f(t, y)$," Journal of 
Computational and Applied Mathematics, vol. 17, no. 3, pp. 363368, 1987.

[11] C. J. Cramer, Essentials of Computational Chemistry, John Wiley \& Sons, Chichester, UK, 2004.

[12] J. M. Franco and M. Palacios, "High-order P-stable multistep methods," Journal of Computational and Applied Mathematics, vol. 30, no. 1, pp. 1-10, 1990 .

[13] L. G. Ixaru and M. Rizea, "Comparison of some four-step methods for the numerical solution of the Schrödinger equation," Computer Physics Communications, vol. 38, no. 3, pp. 329-337, 1985.

[14] L. G. Ixaru and M. Micu, Topics in Theoretical Physics, Central Institute of Physics, Bucharest, Romania, 1978.

[15] L. G. Ixaru and M. Rizea, "A numerov-like scheme for the numerical solution of the Schrödinger equation in the deep continuum spectrum of energies," Computer Physics Communications, vol. 19, no. 1, pp. 23-27, 1980.

[16] F. Jensen, Introduction to Computational Chemistry, John Wiley \& Sons, Chichester, UK, 2007.

[17] Z. Kalogiratou, T. Monovasilis, and T. E. Simos, "New modified Runge-Kutta-Nyström methods for the numerical integration of the Schrödinger equation," Computers \& Mathematics with Applications, vol. 60, no. 6, pp. 1639-1647, 2010.

[18] A. A. Kosti, Z. A. Anastassi, and T. E. Simos, "Construction of an optimized explicit Runge-Kutta-Nyström method for the numerical solution of oscillatory initial value problems," Computerse Mathematics with Applications, vol. 61, no. 11, pp. 3381-3390, 2011.

[19] J. D. Lambert, Numerical Methods for Ordinary Differential Systems. The Initial Value Problem, John Wiley \& Sons, New York, NY, USA, 1991.

[20] A. R. Leach, Molecular Modelling-Principles and Applications, Pearson, Essex, UK, 2001.

[21] T. Lyche, "Chebyshevian multistep methods for ordinary differential equations," Numerische Mathematik, vol. 19, no. 1, pp. 65$75,1972$.

[22] T. Monovasilis, Z. Kalogiratou, and T. E. Simos, "A family of trigonometrically fitted partitioned Runge-Kutta symplectic methods," Applied Mathematics and Computation, vol. 209, no. 1, pp. 91-96, 2009.

[23] T. Monovasilis, Z. Kalogiratou, and T. E. Simos, "Exponentially fitted symplectic Runge-Kutta-Nyström methods," Applied Mathematics \& Information Sciences, vol. 7, no. 1, pp. 81-85, 2013.

[24] G. A. Panopoulos and T. E. Simos, "An eight-step semiembedded predictor-corrector method for orbital problems and related IVPs with oscillatory solutions for which the frequency is unknown," Journal of Computational and Applied Mathematics, vol. 290, pp. 1-15, 2015.

[25] G. A. Panopoulos and T. E. Simos, "An optimized symmetric 8-step semi-embedded predictor-corrector method for IVPs with oscillating solutions," Applied Mathematics \& Information Sciences, vol. 7, no. 1, pp. 73-80, 2013.

[26] G. A. Panopoulos and T. E. Simos, "A new optimized symmetric Embedded Predictor-Corrector Method (EPCM) for initialvalue problems with oscillatory solutions," Applied Mathematics and Information Sciences, vol. 8, no. 2, pp. 703-713, 2014.

[27] D. F. Papadopoulos and T. E. Simos, "A modified RungeKutta-Nyström method by using phase lag properties for the numerical solution of orbital problems," Applied Mathematics \& Information Sciences, vol. 7, no. 2, pp. 433-437, 2013.
[28] D. F. Papadopoulos and T. E. Simos, "The use of phase lag and amplification error derivatives for the construction of a modified Runge-Kutta-Nyström method," Abstract and Applied Analysis, vol. 2013, Article ID 910624, 11 pages, 2013.

[29] G. D. Quinlan and S. Tremaine, "Symmetric multistep methods for the numerical integration of planetary orbits," Astronomical Journal, vol. 100, no. 5, pp. 1694-1700, 1990.

[30] A. D. Raptis and A. C. Allison, "Exponential-fitting methods for the numerical solution of the Schrödinger equation," Physics Communications, vol. 14, no. 1-2, pp. 1-5, 1978.

[31] D. P. Sakas and T. E. Simos, "Multiderivative methods of eighth algebraic order with minimal phase-lag for the numerical solution of the radial Schrödinger equation," Journal of Computational and Applied Mathematics, vol. 175, no. 1, pp. 161-172, 2005.

[32] T. E. Simos, "Dissipative trigonometrically-fitted methods for linear second-order IVP s with oscillating solution," Applied Mathematics Letters, vol. 17, no. 5, pp. 601-607, 2004.

[33] T. E. Simos, "Closed Newton-Cotes trigonometrically-fitted formulae of high order for long-time integration of orbital problems," Applied Mathematics Letters, vol. 22, no. 10, pp. 16161621, 2009.

[34] T. E. Simos, "Exponentially and trigonometrically fitted methods for the solution of the Schrödinger equation," Acta Applicandae Mathematicae, vol. 110, no. 3, pp. 1331-1352, 2010.

[35] T. E. Simos, "New stable closed Newton-Cotes trigonometrically fitted formulae for long-time integration," Abstract and Applied Analysis, vol. 2012, Article ID 182536, 15 pages, 2012.

[36] T. E. Simos, "Optimizing a hybrid two-step method for the numerical solution of the Schrödinger equation and related problems with respect to phase-lag," Journal of Applied Mathematics, vol. 2012, Article ID 420387, 17 pages, 2012.

[37] T. E. Simos, "High order closed Newton-Cotes trigonometrically-fitted formulae for the numerical solution of the Schrödinger equation," Applied Mathematics and Computation, vol. 209, no. 1, pp. 137-151, 2009.

[38] H. Ramos, Z. Kalogiratou, Th. Monovasilis, and T. E. Simos, "An optimized two-step hybrid block method for solving general second order initial-value problems," Numerical Algorithms, vol. 72, no. 4, pp. 1089-1102, 2016.

[39] T. Monovasilis, Z. Kalogiratou, and T. E. Simos, "Construction of exponentially fitted symplectic Runge-Kutta-Nyström methods from partitioned Runge-Kutta methods," Mediterranean Journal of Mathematics, vol. 13, no. 4, pp. 2271-2285, 2016.

[40] T. E. Simos, "On the explicit four-step methods with vanished phase-lag and its first derivative," Applied Mathematics $\alpha$ Information Sciences, vol. 8, no. 2, pp. 447-458, 2014.

[41] T. E. Simos, "Exponentially fitted Runge-Kutta methods for the numerical solution of the Schrödinger equation and related problems," Computational Materials Science, vol. 18, no. 3-4, pp. 315-332, 2000.

[42] S. Stavroyiannis and T. E. Simos, "Optimization as a function of the phase-lag order of nonlinear explicit two-step $P$-stable method for linear periodic IVPs," Applied Numerical Mathematics, vol. 59, no. 10, pp. 2467-2474, 2009.

[43] E. Stiefel and D. G. Bettis, "Stabilization of Cowell's method," Numerische Mathematik, vol. 13, pp. 154-175, 1969.

[44] K. Tselios and T. E. Simos, "Runge-Kutta methods with minimal dispersion and dissipation for problems arising from computational acoustics," Journal of Computational and Applied Mathematics, vol. 175, no. 1, pp. 173-181, 2005. 
[45] C. Tsitouras, I. T. Famelis, and T. E. Simos, "On modified Runge-Kutta trees and methods," Computers \& Mathematics with Applications, vol. 62, no. 4, pp. 2101-2111, 2011.

[46] R. Vujasin, M. Senčanski, J. Radić-Perić, and M. Perić, "A comparison of various variational approaches for solving the one-dimensional vibrational Schrödinger equation," MATCH Communications in Mathematical and in Computer Chemistry, vol. 63, no. 2, pp. 363-378, 2010.

[47] A. D. Raptis and J. R. Cash, "A variable step method for the numerical integration of the one-dimensional Schrödinger equation," Computer Physics Communications, vol. 36, no. 2, pp. 113-119, 1985.

[48] J. R. Dormand, M. E. A. El-Mikkawy, and P. J. Prince, "Families of Runge-Kutta-Nyström formulae," IMA Journal of Numerical Analysis, vol. 7, no. 2, pp. 235-250, 1987.

[49] J. R. Dormand and P. J. Prince, "A family of embedded Runge-Kutta formulae," Journal of Computational and Applied Mathematics, vol. 6, no. 1, pp. 19-26, 1980.

[50] K. Mu and T. E. Simos, "A Runge-Kutta type implicit high algebraic order two-step method with vanished phase-lag and its first, second, third and fourth derivatives for the numerical solution of coupled differential equations arising from the Schrödinger equation," Journal of Mathematical Chemistry, vol. 53, no. 5, pp. 1239-1256, 2015.

[51] H. Ning and T. E. Simos, "A low computational cost eight algebraic order hybrid method with vanished phase-lag and its first, second, third and fourth derivatives for the approximate solution of the Schrödinger equation," Journal of Mathematical Chemistry, vol. 53, no. 6, pp. 1295-1312, 2015.

[52] M. Liang and T. E. Simos, "A new four stages symmetric twostep method with vanished phase-lag and its first derivative for the numerical integration of the Schrödinger equation," Journal of Mathematical Chemistry, vol. 54, no. 5, pp. 1187-1211, 2016.

[53] X. Xi and T. E. Simos, "A new high algebraic order four stages symmetric two-step method with vanished phase-lag and its first and second derivatives for the numerical solution of the Schrödinger equation and related problems," Journal of Mathematical Chemistry, vol. 54, no. 7, pp. 1417-1439, 2016.

[54] Z. Zhou and T. E. Simos, "A new two stage symmetric twostep method with vanished phase-lag and its first, second, third and fourth derivatives for the numerical solution of the radial Schrödinger equation," Journal of Mathematical Chemistry, vol. 54, no. 2, pp. 442-465, 2016.

[55] T. E. Simos, "Multistage symmetric two-step $P$-stable method with vanished phase-lag and its first, second and third derivatives," Applied and Computational Mathematics, vol. 14, no. 3, pp. 296-315, 2015.

[56] R. B. Bernstein, A. Dalgarno, H. Massey, and I. C. Percival, "Thermal scattering of atoms by homonuclear diatomic molecules," Proceedings of the Royal Society A: Mathematical, Physical and Engineering Sciences, vol. 274, no. 1359, pp. 427442, 1963.

[57] R. B. Bernstein, "Quantum mechanical (phase shift) analysis of differential elastic scattering of molecular beams," The Journal of Chemical Physics, vol. 33, no. 3, pp. 795-804, 1960. 


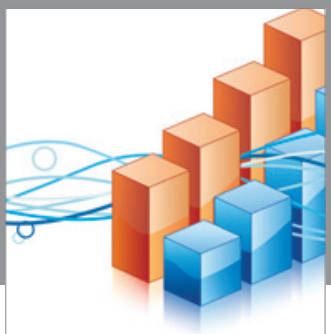

Advances in

Operations Research

vatem alat4

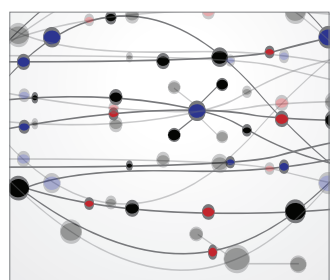

\section{The Scientific} World Journal
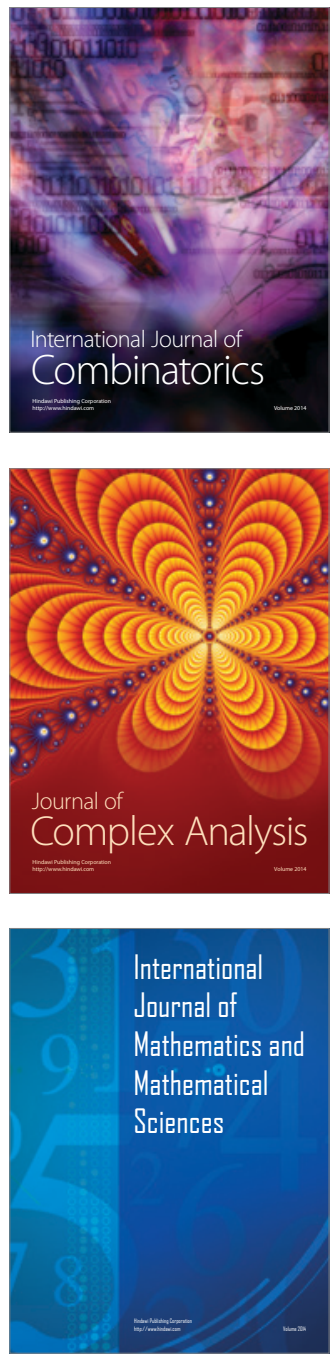
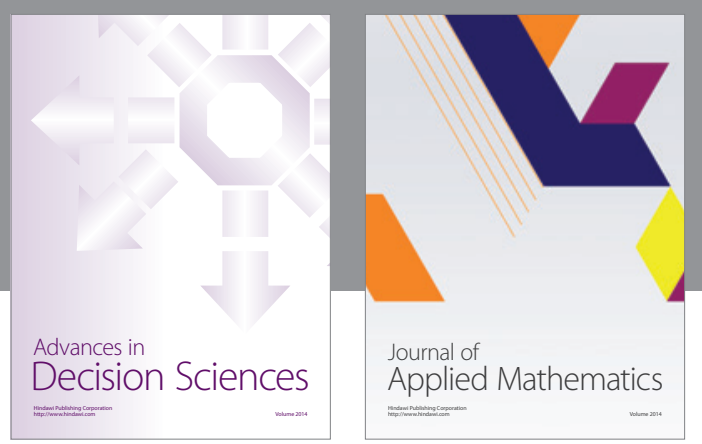

Algebra

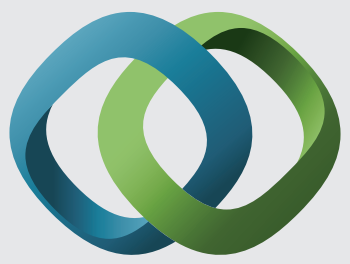

\section{Hindawi}

Submit your manuscripts at

http://www.hindawi.com
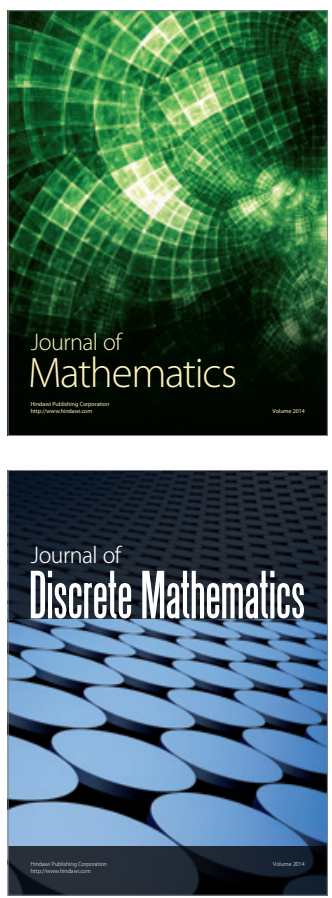

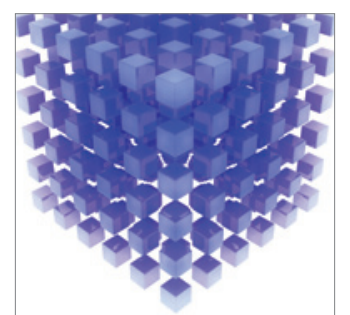

Mathematical Problems in Engineering
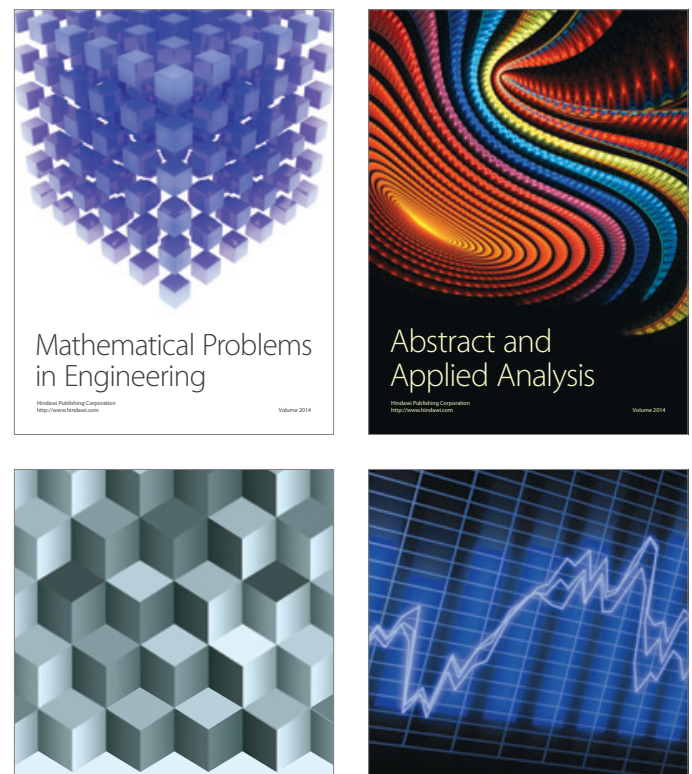

Journal of

Function Spaces

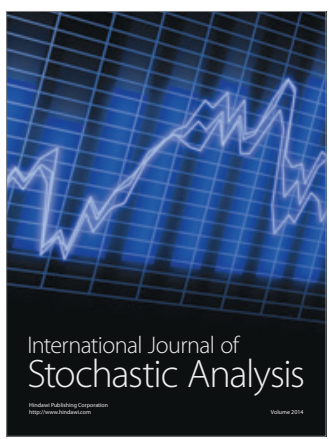

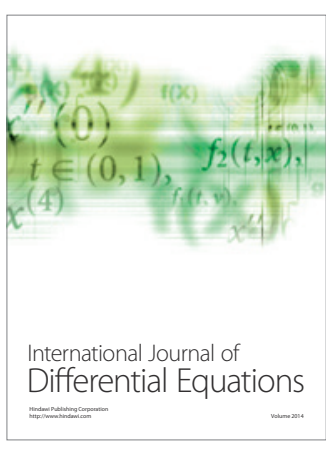
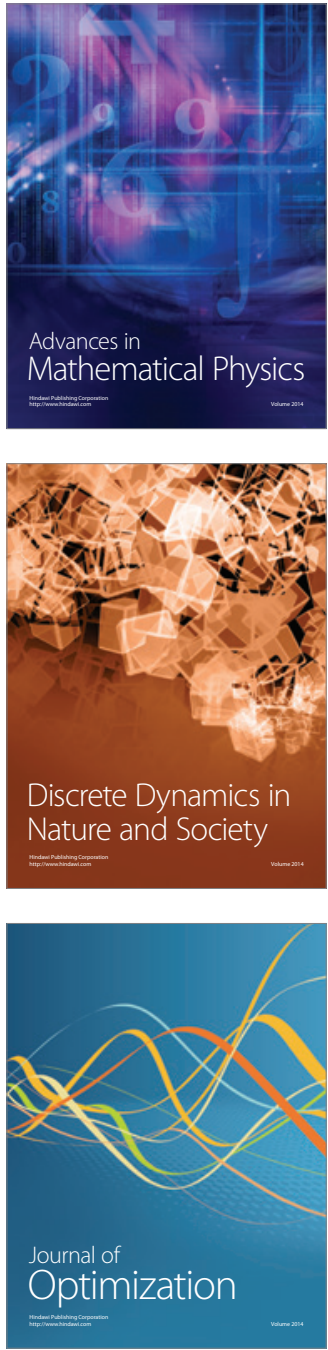